\title{
On the possibility of electrooptic operation by orbital angular momentum of light beams via Pockels effect in crystals
}

\author{
Skab I.P., Vasylkiv Yu.V. and Vlokh R.O. \\ Institute of Physical Optics, 23 Dragomanov St., 79005 Lviv, Ukraine, \\ E-mail:vlokh@ifo.lviv.ua
}

Received: 29.04 .2011

\begin{abstract}
We have shown that some special configurations of electric field created in electrooptic crystals can lead to appearance of orbital angular momentum in the outgoing light beam, provided that the incident circularly polarised beam propagates along the optic axis direction. It has been found that the topological charge of outgoing helical mode is equal to unity. We have also demonstrated on the canonical examples of electrooptic crystals $\mathrm{LiNbO}_{3}$ and $\mathrm{LiTaO}_{3}$ that the efficiency of SAM-toOAM conversion can be gradually operated by the electric field, using the Pockels effect.
\end{abstract}

Keywords: spin angular momentum of light, orbital angular momentum operation, Pockels effect

PACS: $42.50 . \mathrm{Tx}, 78.20 . \mathrm{Jq}$

UDC: 535.012 .2

\section{Introduction}

In recent years, real-time operation by angular momentum of an optical beam has become one of hot topics in quantum optics. The interest to this branch of singular optics [1] is stipulated by novel possibilities for applications of quantum properties of photons, e.g. in quantum computing, cryptography, and even quantum teleportation [2-4]. Possible utilisation of qubits and qudits in processing of information in quantum computers has given rise to increasing information content which can be simultaneously processed. In fact, these possibilities appear if one uses spin and orbital angular momenta of optical beams, which represent quantum quantities. Spin angular momentum (SAM) for the orthogonal circular polarisations of light acquires only two values expressed in the units of $\hbar(s=+1$ and $s=-1$ [5]), while the value of orbital angular momentum (OAM) can, in principle, follow to infinity $(l=0, \pm 1, \pm 2, \ldots)[6]$. Hence, the OAM has some advantages in the information processing, when compare with the SAM, since a single photon has only two distinct spin states and infinitely many distinct OAM states. In such a case the information can be encoded by multiplying a number of distinguishable states, because a photon can carry an arbitrarily large amount of information distributed over its spin and orbital quantum states [7]. A problem which should still be solved for realisation of quantum photonic encoding consists in developing efficient methods for real-time SAM-to-OAM conversion.

It has been shown [8-10] that so-called q-plates which in fact represent liquid-crystalline plates revealing a structural defect in the geometrical centre of the plate, with a topological charge equal to unity, facilitate an efficient SAM-to-OAM conversion performed with the aid of temperature tuning or electrooptic operation of orientation of a director. In case of propagation of nearly plane circular wave through such a plate, the outgoing helical mode acquires the OAM equal to \pm 2 . Notice that liquid crystals have a number of disadvantages, when compared with their 
solid analogues. For instance, they (i) manifest low response speeds due to their viscosity, (ii) are relatively unstable, (iii) cannot be used for operation of powerful laser radiation, and (iv) often reveal unnecessary nonlinear responses.

Recently we have suggested a method of SAM-to-OAM conversion that employs solid crystalline materials subjected to torsion stresses [11,12]. Then the emergent light beam should have the OAM equal to \pm 1 , whereas the topological defect strength associated with optical indicatrix orientation is equal to $\pm 1 / 2$, in terms used for the liquid crystals. As a consequence the OAM quantum number would result in a row of even quantum numbers $l=0, \pm 2, \pm 4 \ldots$ in the case of q-plates with unit topological defects [8] and a row of discrete values $l=0, \pm 1, \pm 2, \ldots$ in the case of solid crystals. Then utilisation of solid crystals should increase twice the number of states in which information can be encoded. On the other hand, piezooptic effect associated with the torsion stresses is rather difficult to realise in practice when designing relevant devices and, moreover, some additional piezoelectric transducers are necessary in order to convert electrical signals to mechanical stresses. Hence, developing of direct, electrically driven, operation of the OAM on the basis of solid crystalline materials represents an important problem. The present work is aimed to solve this problem on the basis of electrooptic Pockels effect in solid crystals.

\section{Basic relations and results of simulations}

As already mentioned, we have earlier demonstrated that a spatial distribution of optical birefringence induced by the torsion stresses possesses a singular point of zero birefringence, which belongs to a torsion axis. In general, coordinate distribution of the torsion-induced birefringence has a conical shape. Due to this distribution, the outgoing wave acquires a helical phase and an OAM. While searching electrooptic analogues of the torsion-induced birefringence distributions, one should proceed from the following requirements: a crystal should be noncentrosymmetric and optically uniaxial, while an electric field should be spatially distributed in a special manner, with a singular value at the line parallel to optic axis $Z$ of a crystal which crosses a geometrical centre of its $X Y$ cross section (here the axes of the coordinate system $X Y Z$ are parallel to the eigenvectors of the Fresnel ellipsoid).

Let us consider a 'conical' spatial distribution of the electric field (see Fig. 1) in crystals that belong to the point symmetry group $3 \mathrm{~m}$ (e.g., $\mathrm{LiNbO}_{3}$ and $\mathrm{LiTaO}_{3}$ crystals). Such a distribution can be produced by two circular electrodes attached to front and back $X Y$ faces of a sample. When the electrodes essentially differ by their radiuses (e.g., the radius of one of them tends to zero), the projections $E_{1}=E_{x}$ and $E_{2}=E_{y}$ of the electric field will appear. The electric field components are determined by relations

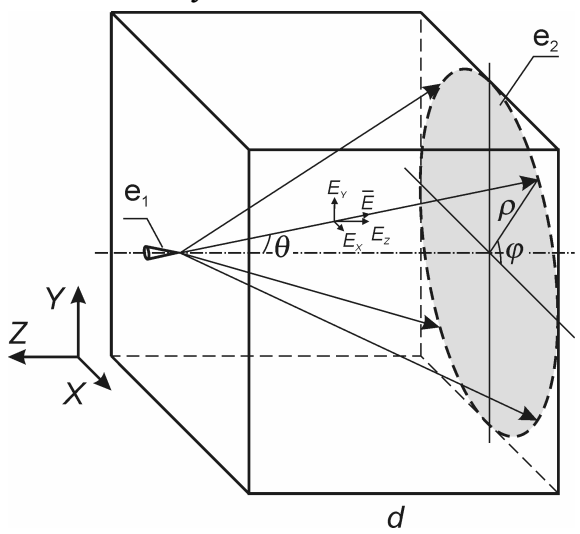

Fig. 1. Schematic view of a crystalline plate with circular electrodes $\mathrm{e}_{1}$ and $\mathrm{e}_{2}$ and a conical spatial distribution of electric field created by those electrodes. 


$$
E_{1}=k X, E_{2}=k Y, E_{3}=k Z,
$$

where

$$
k=\frac{U}{d} \frac{Z}{X^{2}+Y^{2}+Z^{2}} .
$$

In the spherical coordinate system defined by $X=\rho \sin \Theta \cos \varphi, Y=\rho \sin \Theta \sin \varphi$ and $Z=\rho \cos \Theta$ we obtain

$$
\begin{aligned}
& E_{1}=\frac{U}{d} \frac{\tan \Theta}{1+\tan ^{2} \Theta} \cos \varphi, \\
& E_{2}=\frac{U}{d} \frac{\tan \Theta}{1+\tan ^{2} \Theta} \sin \varphi, \\
& E_{3}=\frac{U}{d\left(1+\tan ^{2} \Theta\right)},
\end{aligned}
$$

where $U$ is the applied voltage, $d$ the crystalline plate thickness, and $E_{0}=U / d$. One can see that the $E_{1}$ and $E_{2}$ components are equal to zero when $\Theta=0$ (a case of homogeneous field, with the field lines parallel to the $Z$ axis) and they increase with increasing $\Theta$ and decreasing $d$. Notice that we have neglected the electric field appearing behind the cone limited by the filed lines presented in Fig. 1.

The electrooptic Pockels effect is described by the relation $\Delta B_{i}=r_{i j} E_{j}$, with $r_{i j}$ being the electrooptic tensor components and $\Delta B_{i}$ the increment of optical impermeability tensor. The electrooptic tensor for the point group $3 \mathrm{~m}(m \perp X)$ has the following form:

\begin{tabular}{c|ccc} 
& $E_{1}$ & $E_{2}$ & $E_{3}$ \\
\hline$\Delta B_{1}$ & 0 & $-r_{22}$ & $r_{13}$ \\
$\Delta B_{2}$ & 0 & $r_{22}$ & $r_{13}$ \\
$\Delta B_{3}$ & 0 & 0 & $r_{33}$ \\
$\Delta B_{4}$ & 0 & $r_{51}$ & 0 \\
$\Delta B_{5}$ & $r_{51}$ & 0 & 0 \\
$\Delta B_{6}$ & $-r_{22}$ & 0 & 0
\end{tabular}

Since the optical beam propagates along the $Z$ axis, further on we can consider only the $X Y$ cross section of the Fresnel ellipsoid. The equation of the $X Y$ cross section of optical indicatrix perturbed by the given field configuration reads as

$$
\left(B_{1}-r_{22} E_{2}+r_{13} E_{3}\right) X^{2}+\left(B_{1}+r_{22} E_{2}+r_{13} E_{3}\right) Y^{2}-2 r_{22} E_{1} X Y=1 .
$$

Then the relations for the refractive indices and the induced birefringence are readily obtained as

$$
\begin{gathered}
n_{1,2}=n_{o}-\frac{1}{2} n_{o}^{3}\left(r_{13} E_{3} \pm r_{22} \sqrt{E_{1}^{2}+E_{2}^{2}}\right)=n_{o}-\frac{1}{2} n_{o}^{3} k\left(r_{13} Z \pm r_{22} \sqrt{X^{2}+Y^{2}}\right) \\
\Delta n_{12}=-n_{o}^{3} r_{22} \sqrt{E_{1}^{2}+E_{2}^{2}}=-n_{o}^{3} r_{22} k \sqrt{X^{2}+Y^{2}}, \text { or } \\
\Delta n_{12}=-n_{o}^{3} r_{22} E_{0} \frac{Z \sqrt{X^{2}+Y^{2}}}{X^{2}+Y^{2}+Z^{2}}=-n_{o}^{3} r_{22} E_{0} \frac{\rho^{\prime}}{\rho^{2}} Z
\end{gathered}
$$

where $\rho^{\prime}=\sqrt{X^{2}+Y^{2}}$ is the polar coordinate $\left(X=\rho^{\prime} \cos \varphi\right.$ and $\left.Y=\rho^{\prime} \sin \varphi\right)$ and $n_{o}$ the 
ordinary refractive index.

Contrary to the torsion-induced birefringence $[13,14]$, the birefringence caused by the electric field reveals nonlinear dependences on the both coordinates $X$ and $Y$, which in general are defined by the ratio $\frac{\rho^{\prime}}{\rho^{2}} Z$. However, the dependence of the optical indicatrix rotation angle on the polar angle $\varphi$ is the same as that appearing due to torsion in the crystals of the symmetry group $3 \mathrm{~m}:$

$$
\tan 2 \zeta_{3}=E_{1} / E_{2}=X / Y=\cot \varphi, \text { or } \zeta_{3}=\pi / 4-\varphi / 2 .
$$

The only difference with the optical indicatrix rotation induced by torsion applied around the $Z$ axis is that the initial angle is equal to $\alpha_{0}=\pi / 4$ in the present case, while in the case of the torsion it is equal to zero. As seen from Eqs. (10), the angle of the optical indicatrix rotation depends on neither the $Z$ coordinate nor the electrooptic coefficient. One can easily simulate this angular dependence, which should be the same for all of the crystals belonging to the symmetry group 3m (see Fig. 2).

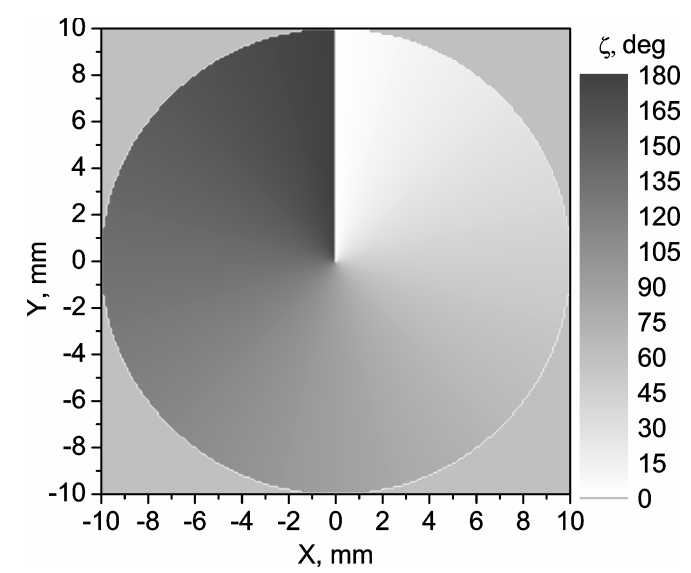

Fig. 2. Spatial distribution of electrooptically induced optical indicatrix rotation for the crystals that belong to the point symmetry group $3 \mathrm{~m}$.

When considering the central point of the $X Y$ cross section as a defect on the spatial map of optical indicatrix orientation and using definitions proper for the liquid crystals, one can see that the topological strength of this defect is equal to $\mathrm{q}= \pm 1 / 2$ and the angle $\zeta_{3}$ amounts to half the angle $\varphi$. It is obvious that the sign of the optical indicatrix rotation would reverse whenever the sign of the applied electric field does so. This corresponds to a change in the sign of the induced birefringence and a rotation of the optical indicatrix by $90 \mathrm{deg}$. In fact, $\Delta n_{12}$ has opposite signs when, e.g. $E_{1}$ change the sign, while $E_{2}=0$ (see Eq. (9)).

Now let us analyse spatial distribution of the phase difference induced in the $X Y$ plane. Since the birefringence depends on all the coordinates $X, Y$ and $Z$, it is convenient to divide a sample by $n$ homogeneous layers perpendicular to the $Z$ direction. In its turn, each of the layers is divided by $i \times j$ homogeneous elementary cells in the $X Y$ plane. In practice, we have used the division given by $i=200, j=200$, and $n_{\max }=1 \div 100$. One can use a standard Jones matrix approach for simulating the electrically induced phase difference. The resulting Jones matrix may be written as

$$
J^{i j}=\prod_{n=1}^{n_{\max }} J_{n}^{i j}
$$

where $J_{n}^{i j}$ are the matrices of the homogeneous elementary cells given by

$$
J_{n}^{i j}=\left|\begin{array}{cc}
\left(e^{i \Delta \Gamma_{n}^{i j} / 2} \cos ^{2} \zeta_{n}^{i j}+e^{-i \Gamma_{n}^{i j} / 2} \sin ^{2} \zeta_{n}^{i j}\right) & i \sin \left(\Delta \Gamma_{n}^{i j} / 2\right) \sin 2 \zeta_{n}^{i j} \\
i \sin \left(\Delta \Gamma_{n}^{i j} / 2\right) \sin 2 \zeta_{n}^{i j} & \left(e^{i \Delta \Gamma_{n}^{i j} / 2} \sin ^{2} \zeta_{n}^{i j}+e^{-i \Delta \Gamma_{n}^{i j} / 2} \cos ^{2} \zeta_{n}^{i j}\right)
\end{array}\right|,
$$


while $\Delta \Gamma_{n}^{i j}=\frac{2 \pi d_{n}^{i j}}{\lambda}\left\{-n_{0}^{3} r_{22} \frac{U}{d} \frac{Z \sqrt{X^{2}+Y^{2}}}{X^{2}+Y^{2}+Z^{2}}\right\} \quad$ and $\quad \zeta_{n}^{i j}=\frac{1}{2} \arctan \frac{X}{Y} \quad$ represent the phase difference and the angle of optical indicatrix rotation within the elementary cells, respectively, and $d_{n}^{i j}$ is the thickness of the cell along the direction of light propagation. The resulting phase difference for each of the elementary beams that crosses the $i \times j$ array becomes as follows:

$$
\Delta \Gamma^{i j}=2 \arccos \left(\operatorname{Re}\left[J^{i j}\right]_{p p}\right),
$$

where the subscript $p p$ corresponds to diagonal components of the Jones matrix. The $X Y$ maps of the phase difference thus obtained for $\mathrm{LiNbO}_{3}$ and $\mathrm{LiTaO}_{3}$ crystals are shown in Fig. 3. In our simulations, we have used the following data: $d=5 \mathrm{~mm}$, the electrode radius of $R=10 \mathrm{~mm}$, $r_{22}=3.4 \times 10^{-12} \mathrm{~m} / \mathrm{V}, \quad n_{o}=2.286$ for $\mathrm{LiNbO}_{3}[15], \quad r_{22}=20.0 \times 10^{-12} \mathrm{~m} / \mathrm{V}, \quad n_{o}=2.176$ for $\mathrm{LiTaO}_{3}$ crystals [16], and $\lambda=632.8 \mathrm{~nm}$.
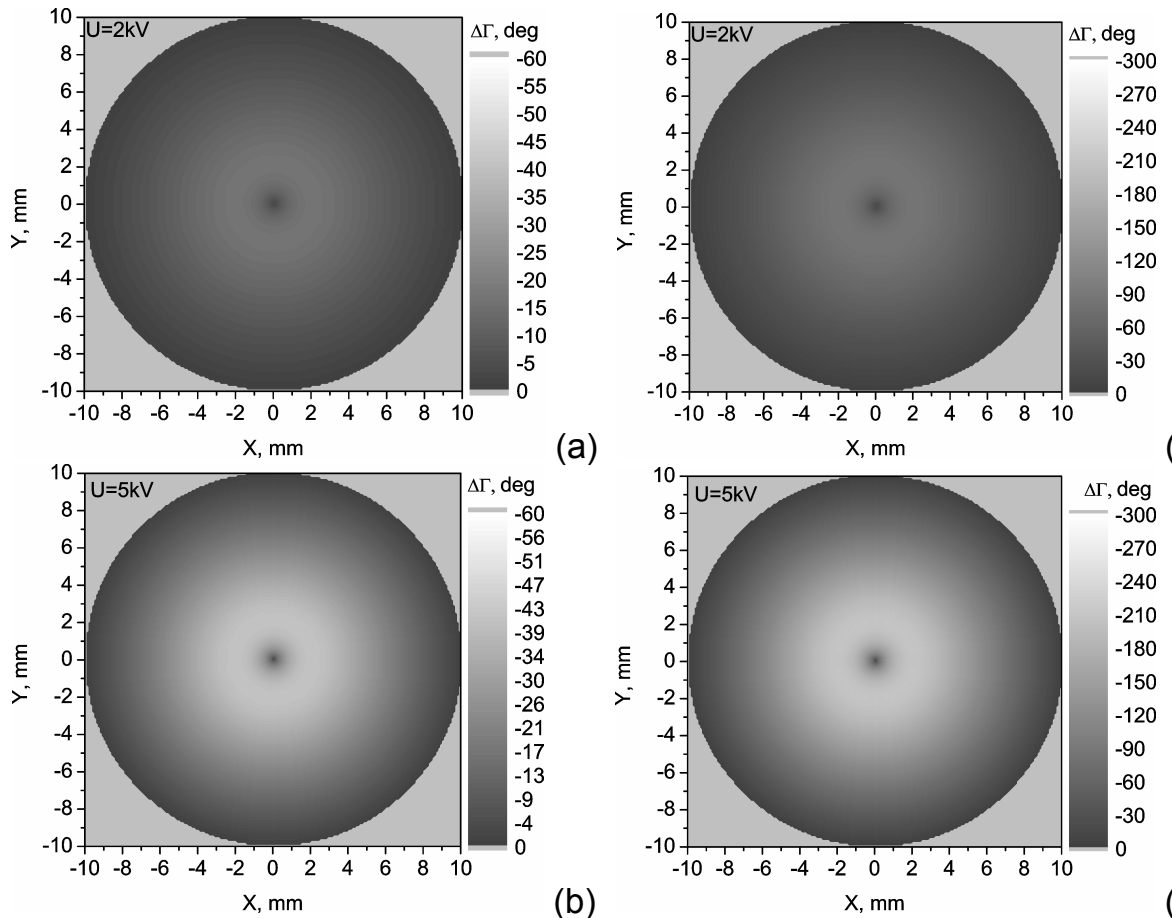

(d)
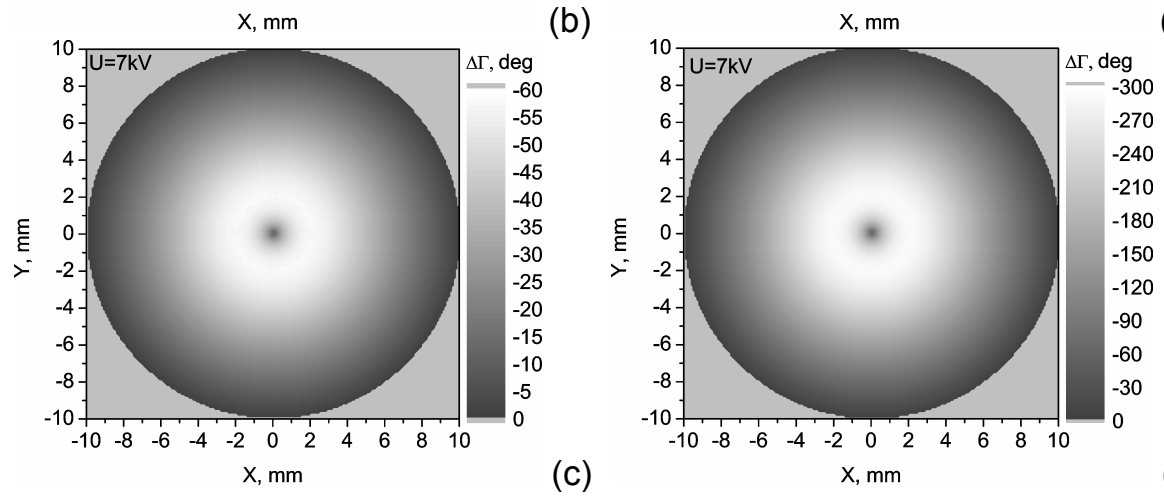

(e)

Fig. 3. $X Y$ maps of phase difference induced by the electrical voltages $2 \mathrm{kV}(\mathrm{a}, \mathrm{d}), 5 \mathrm{kV}(\mathrm{b}, \mathrm{e})$ and $7 \mathrm{kV}(\mathrm{c}, \mathrm{f})$ in $\mathrm{LiNbO}_{3}(\mathrm{a}, \mathrm{b}, \mathrm{c})$ and $\mathrm{LiTaO}_{3}(\mathrm{~d}, \mathrm{e}, \mathrm{f})$ crystals. 
As seen from Fig. 3, the induced phase difference reveals a circular distribution in the $X Y$ plane, being equal to zero at the geometrical centre of the $X Y$ cross section. A typical spatial distribution of the phase difference along the $X$ axis is presented in Fig. 4.

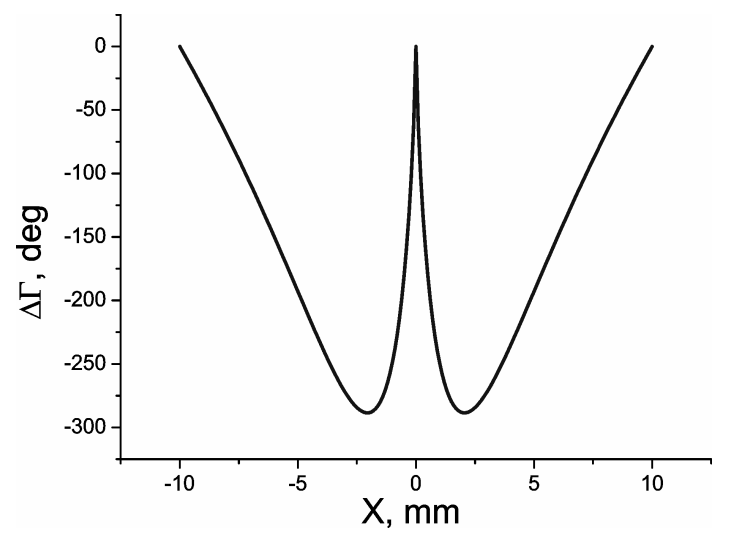

Fig. 4. Dependence of phase difference induced by the electrical voltage $7 \mathrm{kV}$ on the $X$ coordinate for $\mathrm{LiTaO}_{3}$ crystals.
As evident from Fig. 4, the module of the phase difference increases with increasing $|X|$ coordinate, following from a zero value at $X=0$ to its maximum at $X_{m}= \pm 2.06 \mathrm{~mm}$. Note that the $X_{m}$ value is the same for all the cases presented in Fig. 3, being independent of either the electrooptic figure of merit or the electrical voltage applied. Further increase in $X$ leads to decrease of the induced phase difference to zero at $|X|=R=10 \mathrm{~mm}$. The coordinate dependence of the phase difference mentioned above is caused by the two mechanisms: (i) increase in $|\Delta \Gamma|$ occurring with increasing $|X|$, due to increasing $E_{1}$ and $E_{2}$ projections, and (ii) decrease in $|\Delta \Gamma|$ occurring with increasing $|X|$, due to decreasing effective optical path (i.e., the optical path in that part of crystal which is subjected to the electric field).

In order to analyse this dependence in a more detail, one should derive the appropriate analytical relationship. We have found that the mean value of the function describing the phase difference,

$$
\Delta \Gamma=-\frac{2 \pi}{\lambda} n_{o}^{3} r_{22} E_{0} \rho^{\prime} \int_{\frac{\rho^{\prime} d}{R}}^{d} \frac{Z}{\rho^{\prime 2}+Z^{2}} d Z=-\frac{\pi}{\lambda} n_{o}^{3} r_{22} E_{0} \rho^{\prime} \ln \frac{\left(\rho^{\prime 2}+d^{2}\right) R^{2}}{\left(R^{2}+d^{2}\right) \rho^{\prime 2}},
$$

result in a distribution of phase difference in the $X Y$ plane, which is the same as in case of Jones matrix calculation. As a consequence, using the minimising procedure,

$$
\frac{\partial \Delta \Gamma}{\partial \rho^{\prime}}=-\frac{\pi n_{0}^{3} r_{22} E_{0}}{\lambda}\left(\ln \left(\frac{R^{2}\left(\rho^{\prime 2}+d^{2}\right)}{\rho^{\prime 2}\left(R^{2}+d^{2}\right)}\right)-\frac{2 d^{2}}{\rho^{\prime 2}+d^{2}}\right)=0
$$

and solving the equation

$$
\ln \left(\frac{R^{2}\left(\rho^{\prime 2}+d^{2}\right)}{\rho^{\prime 2}\left(R^{2}+d^{2}\right)}\right)-\frac{2 d^{2}}{\rho^{\prime 2}+d^{2}}=0,
$$

yield the relation for the radius that corresponds to the extreme $\Delta \Gamma$ value:

$$
\rho_{\max }^{\prime}=d \frac{\sqrt{-W\left(-\frac{2 R^{2} e^{-2}}{R^{2}+d^{2}}\right)\left(W\left(-\frac{2 R^{2} e^{-2}}{R^{2}+d^{2}}\right)+2\right)}}{W\left(-\frac{2 R^{2} e^{-2}}{R^{2}+d^{2}}\right)+2},
$$


where $W\left(-\frac{2 R^{2} e^{-2}}{R^{2}+d^{2}}\right)$ implies the Lambert $W$ function.

Solving Eq. (17) numerically, we have found that the radius corresponding to the maximum phase difference module is equal to $\rho^{\prime}=2.06 \mathrm{~mm}$ for $R=10 \mathrm{~mm}$ and $d=5 \mathrm{~mm}$. This value agrees well with that obtained on the basis of Jones matrix calculations. Under these conditions (e.g., for $\mathrm{LiTaO}_{3}$ crystals and $U=7 \mathrm{kV}$ ), the phase difference calculated using Eq. (14) is equal to $\Delta \Gamma_{\max }=-288.9 \mathrm{deg}$. Again, it is perfectly correlated with that calculated with the Jones matrix approach.

Using Eqs. (14) and (17), we have computed dependences of the maximum phase difference appearing at the radius $\rho_{\max }^{\prime}$ on the radius $R$ and the thickness $d$, along with the dependences of $\rho_{\max }^{\prime}$ parameter on the radius $R$ and the thickness $d$ (see Fig. 5). The phase difference $\Delta \Gamma_{\max }$ increases with decreasing distance $d$ and radius $R$, while the parameter $\rho_{\max }^{\prime}$ increases with increasing $d$ and $R$. It follows from these results that the complete conversion of a right-handed incident light into a left-handed outgoing one at $\rho_{\max }^{\prime}$ would occur when the induced phase difference equals to $\Delta \Gamma_{\max }=-180 \mathrm{deg}$. The minimal voltage needed for inducing this phase difference under the condition of typical radius of laser beam equal to $\rho_{\max }^{\prime}=1.5 \mathrm{~mm}$ corresponds to the sample thickness of $d=3.4 \mathrm{~mm}$, and the electrode radius of $R=10 \mathrm{~mm}$. This voltage is equal to $4.083 \mathrm{kV}$ and $\sim 20 \mathrm{kV}$ for $\mathrm{LiTaO}_{3}$ and $\mathrm{LiNbO}_{3}$ crystals, respectively.

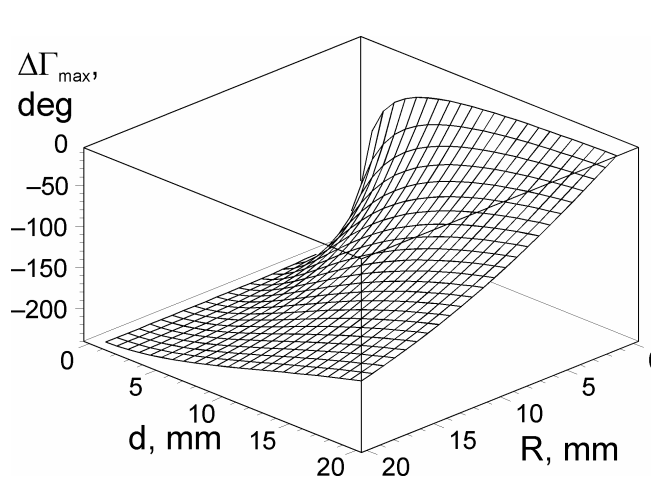

(a)

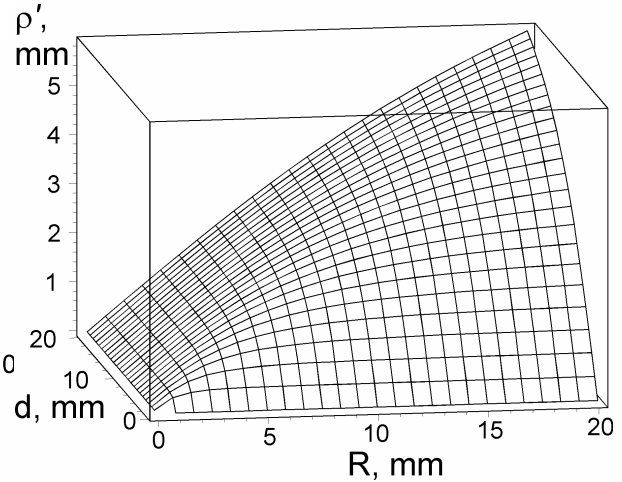

(b)

Fig. 5. Dependences of maximum phase difference appearing at the radius $\rho_{\max }^{\prime}$ on the radius $R$ and the thickness $d(\mathrm{a})$, and dependences of $\rho_{\max }^{\prime}$ parameter on the radius $R$ and the thickness $d(\mathrm{~b})$.

Finally, let us analyse the appearance of the OAM in the emergent light beam having passed through an optical system that consists of a right-handed circular polariser, a crystalline sample subjected to the electric field of conical configuration, and a left-handed circular polariser. Notice that the incident beam can be represented by nearly plane wave, with the beam radius equal to $\rho^{\prime}=R$ and the SAM equal to $S^{i n c}=-\hbar$. The electric field of the emergent light can be written as

$$
E^{\text {out }}\left(\rho^{\prime}, \varphi\right)=E_{a} \cos \frac{\Delta \Gamma\left(\rho^{\prime}\right)}{2}\left[\begin{array}{c}
1 \\
\pm i
\end{array}\right]+i E_{a} \sin \frac{\Delta \Gamma\left(\rho^{\prime}\right)}{2} e^{ \pm i 2 q \varphi \pm i 2 \alpha_{0}}\left[\begin{array}{c}
1 \\
\mp i
\end{array}\right],
$$

where $2 q=m=1$ is the helicity number and $E_{a}$ the wave amplitude. The first term in Eq. (18) 
describes the plane wave with the same SAM as in the incident one (i.e., $-\hbar$ ), while the second summand - the wave with the helical wavefront which carries the OAM (see [17]). Taking into account that the angular momentum must be conserved, one can write the following relation for the SAM-to-OAM conversion:

$$
J^{\text {inc }}=J^{\text {out }}+M
$$

where $J^{i n c}=S^{i n c}=-\hbar$ is the total angular momentum of the incident photon, $J^{\text {out }}=L^{\text {out }}+S^{\text {out }}=-2 q \hbar+\hbar=0$ the total angular momentum of the emergent photon ( $S^{\text {out }}=+\hbar, L^{\text {out }}=-2 q \hbar$ ), and $L^{\text {out }}$ the OAM of the emergent photon. Thus, the mechanical angular momentum transferred to the crystalline sample due to the Beth effect is equal to $M=-\hbar$. However, this relation which reflects the fact of conservation of the angular momentum has been written for the condition $\Delta \Gamma=\pi$, though the phase difference depend on the module $\rho^{\prime}$. In this case one should take into account that the plane wave described by the first term of Eq. (18), with the SAM equal to $-\hbar$, also emerges from the sample. Then the efficiency of the SAM-to-OAM conversion can be defined by a ratio

$$
\eta=\frac{I_{l}^{\text {out }}}{I_{r}^{i n c}} .
$$

where $I_{r}^{\text {inc }}$ is the intensity of the right-handed incident wave and $I_{l}^{\text {out }}$ the intensity of the lefthanded outgoing wave. The $X Y$ intensity distribution for the outgoing beam can be calculated using the Jones matrices (for more details see Ref. [11]). The appropriate spatial intensity distributions calculated for different voltages are presented in Fig. 6, while the dependences of the efficiency $\eta$ on the electrical voltage are given in Fig. 7. It is seen from Fig. 7 that the efficiency reaches $\sim 30 \%$ at $\sim 4 \mathrm{kV}$ for $\mathrm{LiTaO}_{3}$ crystals and at $\sim 20 \mathrm{kV}$ for $\mathrm{LiNbO}_{3}$ crystals. This efficiency can be further increased by decreasing the light beam radius.
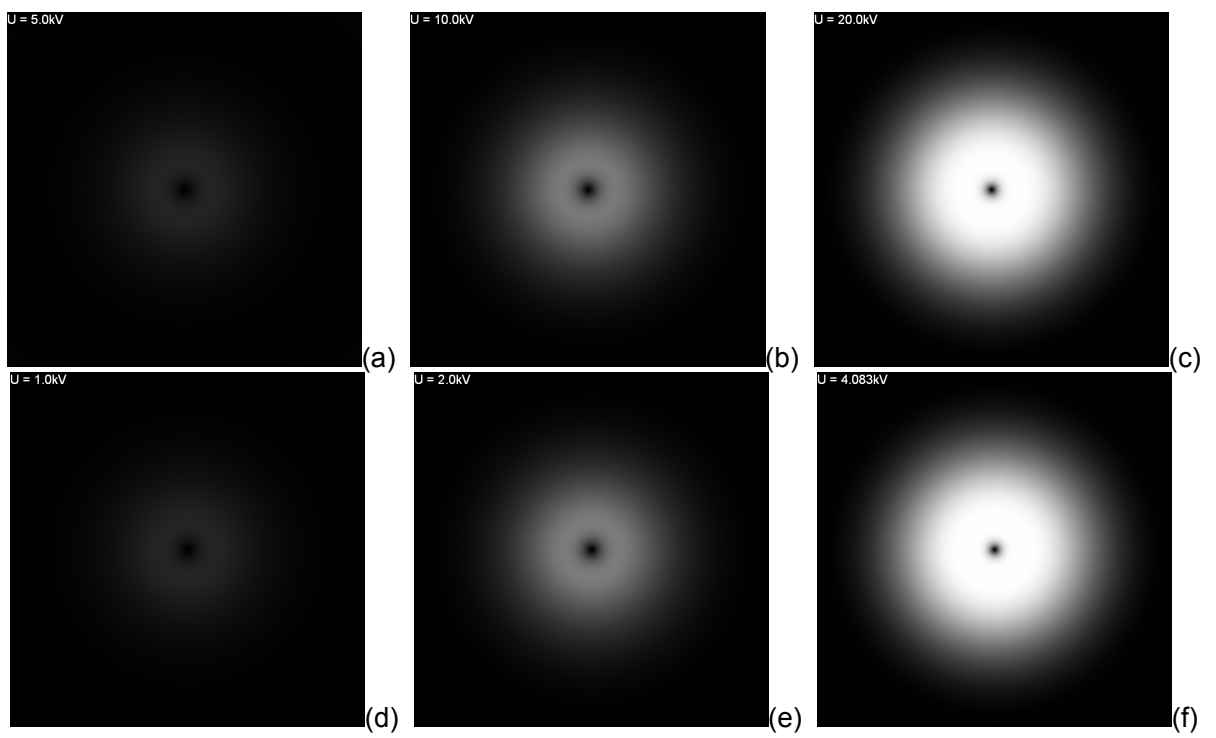

Fig. 6. Spatial distributions of intensity for the beam of $1.5 \mathrm{~mm}$ radius emergent from the system consisting of orthogonal circular polarisers and a sample of $\mathrm{LiNbO}_{3}(\mathrm{a}-5.0 \mathrm{kV}, \mathrm{b}-10.0 \mathrm{kV}$, and c $-20.0 \mathrm{kV})$ or $\mathrm{LiTaO}_{3}$ $(\mathrm{d}-1.0 \mathrm{kV}, \mathrm{e}-2.0 \mathrm{kV}$, and $\mathrm{f}-4.083 \mathrm{kV})$ in between. 

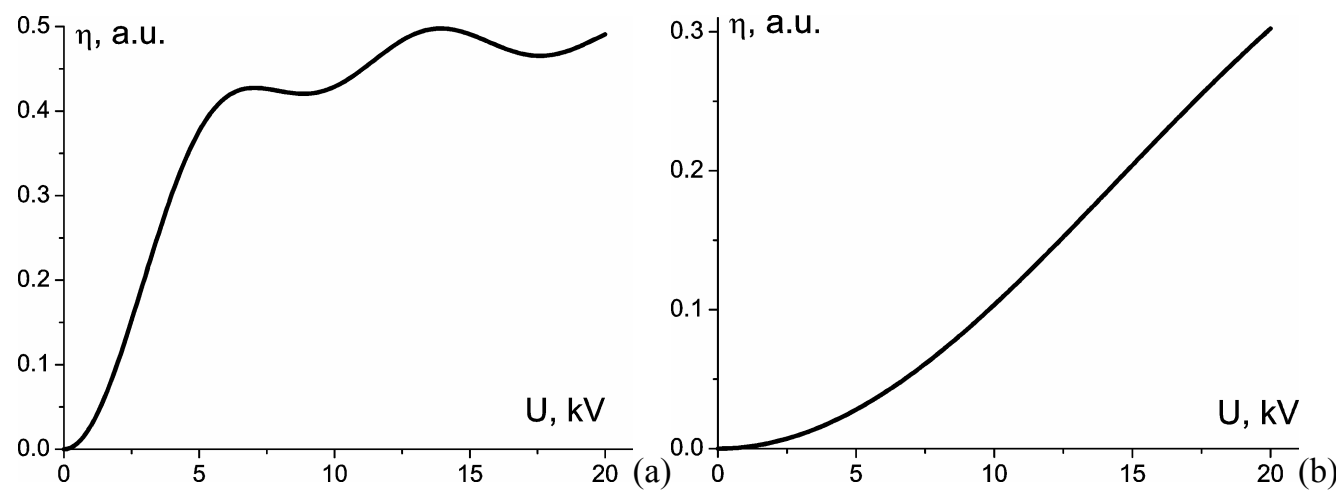

Fig. 7. Dependences of efficiency of SAM-to-OAM conversion on the electrical voltage applied to $\mathrm{LiTaO}_{3}(\mathrm{a})$ and $\mathrm{LiNbO}_{3}$ (b) crystals.

\section{Conclusions}

In this work we have performed computer simulations of the Pockels effect taking place in trigonal crystals of the point symmetry group $3 \mathrm{~m}$, which is caused by a 'conical' configuration of electric field. We have shown that such a field leads to appearance of OAM in the outgoing light beam, whenever the incident circularly polarised beam propagates along the optic axis direction. The corresponding theoretical relations describing spatial distribution of the birefringence and the angle of optical indicatrix rotation have been found. It has been demonstrated that the topological charge of the outgoing helical mode in this system is equal to unity. Using the examples of canonical electrooptic crystals $\mathrm{LiNbO}_{3}$ and $\mathrm{LiTaO}_{3}$, we have revealed that the efficiency of SAMto-OAM conversion can be gradually operated, owing the Pockels effect, by changing the electric field applied to a sample.

\section{References}

1. Soskin M S and Vasnetsov M V, 2001. Singular optics. Progr. Opt. 42: 219-276.

2. David P DiVincenzo, 1995. Quantum computation. Science. 270: 255-261.

3. Kilin S Ya, 1999. Quantum information. Phys. - Uspekhi. 42: 435-452.

4. Boschi D, Branca S, De Martini F, Hardy L and Popescu S, 1998. Experimental realization of teleporting an unknown pure quantum state via dual classical and Einstein-Podolsky-Rosen channels. Phys. Rev. Lett. 80: 1121-1125.

5. Beth R A, 1936. Mechanical detection and measurement of the angular momentum of light. Phys. Rev. 50: 115-125.

6. Allen L, Beijersbergen M W, Spreeuw R J C and Woerdman J P, 1992. Orbital angular momentum of light and the transformation of Laguerre-Gaussian laser modes. Phys. Rev. A. 45: 8186-8189.

7. Gabriel Molina-Terriza, Juan P Torres and Lluis Torner, 2001. Management of the angular momentum of light: preparation of photons in multidimensional vector states of angular momentum. Phys. Rev. Lett. 88: 013601.

8. Ebrahim Karimi, Bruno Piccirillo, Eleonora Nagali, Lorenzo Marrucci and Enrico Santamato, 2009. Efficient generation of orbital angular momentum eigenmodes of light by thermally tuned q-plates. Appl. Phys. Lett. 94: 231124.

9. Bruno Piccirillo, Vincenzo D’Ambrosio, Sergei Slussarenko, Lorenzo Marrucci and Enrico 
Santamato, 2010. Photon spin-to-orbital angular momentum conversation via an ellectrically tunable q-plate. Appl. Phys. Lett. 97: 241104.

10. Marrucci L, Manzo C and Paparo D, 2006. Optical spin-to-orbital angular momentum conversion in inhomogeneous anisotropic media. Phys. Rev. Lett. 96: 163905.

11. Skab I, Vasylkiv Yu, Savaryn V and Vlokh R, 2011. Optical anisotropy induced by torsion stresses in $\mathrm{LiNbO}_{3}$ crystals: appearance of an optical vortex. J. Opt. Soc. Amer. A. 28: 633640 .

12. Ihor Skab, Yurij Vasylkiv, Bohdan Zapeka, Viktoriya Savaryn, and Rostyslav Vlokh, 2011. Appearance of singularities of optical fields under torsion of crystals containing threefold symmetry axes. J. Opt. Soc. Amer. A. 28: 1331-1340.

13. Skab I, Vasylkiv Yu, Savaryn V and Vlokh R, 2010. Relations for optical indicatrix parameters in the conditions of crystal torsion. Ukr. J. Phys. Opt. 11: 193-240.

14. Vasylkiv Yu, Savaryn V, Smaga I, Skab I and Vlokh R, 2010. Determination of piezooptic coefficient $\pi_{14}$ of $\mathrm{LiNbO}_{3}$ crystals under torsion loading. Ukr. J. Phys. Opt. 11: 156-164.

15. http://www.almazoptics.com/LiNbO3.htm

16. http://www.almazoptics.com/LiTaO3.html

17. Marrucci L, 2008. Generation of helical modes of light by spin-to-orbital angular momentum conversion in inhomogeneous liquid crystals. Mol.Cryst.Liq.Cryst. 488: 148-162.

Skab I.P., Vasylkiv Yu.V. and Vlokh R.O., 2011. On the possibility of electrooptic operation by orbital angular momentum of light beams via Pockels effect in crystals Ukr.J.Phys.Opt. 12: $127-136$.

Анотація. В роботі показано, щз певна конфігурація електричного поля, створена в електрооптичному кристалі може приводити до утворення орбітального кутового моменту вихідного променя, якщо падаючий оптичний промінь володіє циркулярною поляризацією і поширюється вздовж оптичної осі кристалу. При цзьому встановлено, щуо топологічний заряд вихідної гелікоїдальної моди дорівнює одиниці. На прикладі відомих електрооптичних кристалів таких як $\mathrm{LiNbO}_{3}$ i $\mathrm{LiTaO}_{3}$ показано, щчо ефективність перетворення спінового кутового моменту в орбітальний кутовий момент може поступово змінюватись під дією електричного поля з використанням ефекту Покельса. 